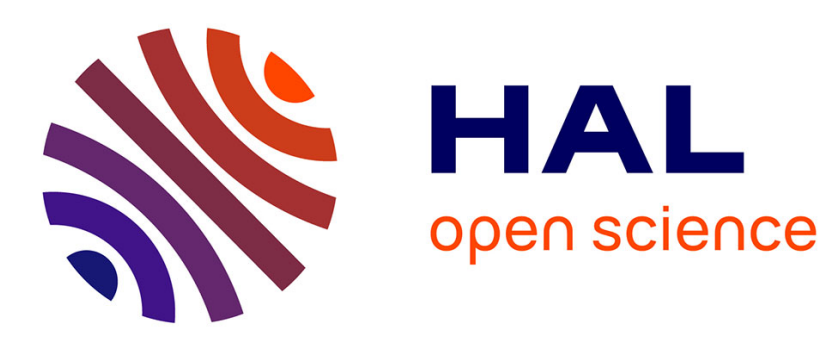

\title{
Evaluation of wearable Kinematic Algorithms for the Monitoring of Ecological Activity
}

\author{
Norbert Noury, Bruno Perriot
}

\section{To cite this version:}

Norbert Noury, Bruno Perriot. Evaluation of wearable Kinematic Algorithms for the Monitoring of Ecological Activity. IEEE International Conference on E-health Networking, Application \& Services, Sep 2018, Ostrava, Czech Republic. hal-01963572

\section{HAL Id: hal-01963572 https://hal.science/hal-01963572}

Submitted on 21 Dec 2018

HAL is a multi-disciplinary open access archive for the deposit and dissemination of scientific research documents, whether they are published or not. The documents may come from teaching and research institutions in France or abroad, or from public or private research centers.
L'archive ouverte pluridisciplinaire HAL, est destinée au dépôt et à la diffusion de documents scientifiques de niveau recherche, publiés ou non, émanant des établissements d'enseignement et de recherche français ou étrangers, des laboratoires publics ou privés. 


\title{
Evaluation of wearable Kinematic Algorithms for the Monitoring of Ecological Activity
}

\author{
Norbert Noury, Bruno Perriot
}

\begin{abstract}
Chronic Obstructive Pulmonary Disease (COPD) causes severe dyspnea during physical exercises. In order to detect a reduction in the intensity of physical activities of COPD subjects, we monitored their physical activity during intensive physical exercises as well as during normal daily activities. A field experiment was performed on 13 COPD patients over periods of 8 hours. Our classifier detects static postures (standing, sitting, lying) with sensitivities 77-94\% and specificities $86-91 \%$.
\end{abstract}

Index Terms-COPD patients, Actimetry, Body postures, Body Sensor Network, Bluetooth.

\section{INTRODUCTION}

$\mathrm{T}_{\mathrm{D}}^{\mathrm{h}}$ he main expression of Chronic Obstructive Pulmonary

Disease (COPD) is airflow reduction. This is a major pathology with a worldwide prevalence of $10 \%$ in adults over 40 [1]. COPD can cause severe dyspnea, especially during physical exercises [2]. It is worse with age. Therefore, a reduction in the intensity of physical exercises and the level of daily activities (walking time, standing or resting time) is well correlated with the severity of the disease and with the risk for future acute exacerbation [3][4]. The "six-minute walk test" [5] is the reference for evaluating the progression of the disease, as well as to evaluate the efficiency of treatments like pulmonary rehabilitation and oxygen therapy. During the "six-minute walk test", the patient walks the longest distance he can, while his blood saturation is monitored with a pulse oximeter. Other similar tests are based on the monitoring of Sp02 during a physical exercise, for instance the "stand up from a chair". This last exercise consists in a 3 minutes series of "standing-sitting down" on a chair, the 1 st minute at a fixed pace and the next 2 minutes at the patient's maximal pace.

Thus, there is growing interest in designing activity monitors to assess objectively the level of everyday physical activity in COPD subjects. Such an "actimeter" should be able to provide details on everyday physical activity - e.g. postures lying, sitting, standing, but also the distance of walking. It must also have additional capability to communicate with other external Bluetooth sensors - e.g. blood pressure sensor, photo plethysmograph, airflow meter,

B. Perriot and Prof. N. Noury are with University of Lyon, Institute of Nanotechnologies of Lyon, France (norbert.noury@univ-lyon1.fr). etc. - to provide a fully comprehensive assessment of the patient's condition.

Monitoring patients in their daily life needs the system to be capable of being used autonomously by the patient himself in his own environment. Some activity monitors have been proposed in the literature [6] for the monitoring of COPD patients, but they are not networked. We therefore developed our IMU with robust embedded algorithm, which can be wirelessly interconnected to medical sensors, for acquiring data on both activity and physiologic, for the monitoring in daily life or in supervised medical exams [7].

\section{MATERIAL AND METHODS}

\section{A. The inertial monitoring unit ACOR+}

The actimeter $(A C O R+\circledR$, Sleepinnov Technology France) is worn on the hip during the day ( Figure 1), and on the sternum during the night.

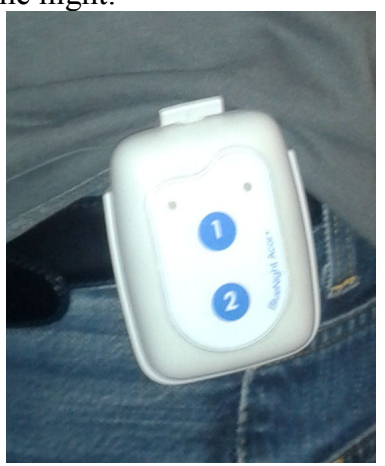

Figure 1. The ACOR+ is an IMU with reduced dimensions $(77 \times 57 \times 25 \mathrm{~mm})$ and light weight $(70 \mathrm{~g})$ to be clipped on the belt.

The ACOR+ can be controlled manually, using the pushbuttons placed on the actimeter, or via a Bluetooth connection from a master device. The Bluetooth connection allows a local master to collect the data and to program ACOR + to perform several exams in a row. The Bluetooth protocol was selected as it is already widely-adopted in several medical sensors available on the market and it allows deploying a local WBAN.

The ACOR+ combines a tri-axis accelerometer (MMA7455, Freescale), for raw data acquisition, a powerful micro- 
controller (STR710, STMicroelectronics) for signal processing and a 2 Go SD-card to store the acquired data. The maximum resolution of the accelerometer is 10 bits, the sensitivity ranges from $2 \mathrm{G}$ to $8 \mathrm{G}$ (4 to $16 \mathrm{mG}$ ). We set the sampling rate to $50 \mathrm{~Hz}$ which corresponds to human actimetry frequencies [8].

The ARM7TDMI micro-controller is operated at $48 \mathrm{MHz}$; it has a $256 \mathrm{~KB}$ program Flash memory and $64 \mathrm{~KB}$ data memory. The ACOR+ reaches an autonomy of 30 hours (@ $50 \mathrm{~Hz}$ ) ; it is adapted to daily monitoring at home.

\section{B. Embedded Algorithms}

The ACOR+ has on-board robust autonomous algorithms to label the postures [9]. It generates a XML report after each exam, which contains the time spent in each postures, the number of steps each day, and stores it on the SD-card.

1) Detection of Active-Inactive states

The accelerometer signals shows greater variability during standing periods than during sitting or lying, because of walking and transfers. Therefore, the first stage of our algorithm computes the variability on the signal of vertical acceleration $x$,

$$
V(n)=\sum_{i=n-\frac{W}{2}}^{i=n+\frac{W}{2}}|x(i)-x(i-1)|
$$

With $W$ the temporal duration of the observation window. When the variability $(V)$ exceeds a predefined threshold $(T h)$ our algorithm labels a pattern of activity.

Actually the variability of the signal during real life sequences shows a background noise $(V=180)$. The peaks correspond to patterns of activity. We computed the probability that a given period belongs to an active or inactive episode, with varying variability $V$ (Figure 2 ). With $T h=335$ we obtained $95 \%$ of episodes correctly attributed to active periods.

\section{2) Discrimination between sitting and lying}

To discriminate the 2 positions of lying and sitting, we computed a criterion of verticality on the low-pass filtered the cut-off frequency is $1 \mathrm{~Hz}$ - accelerometer signals:

$$
\text { Verticality criterion }=\mathrm{X}^{2} /\left(\mathrm{Y}^{2}+\mathrm{Z}^{2}\right)
$$

With $X, Y$ and $Z$, the acceleration signals on the directions vertical, antero-posterior and medio-lateral respectively (Figure 1).

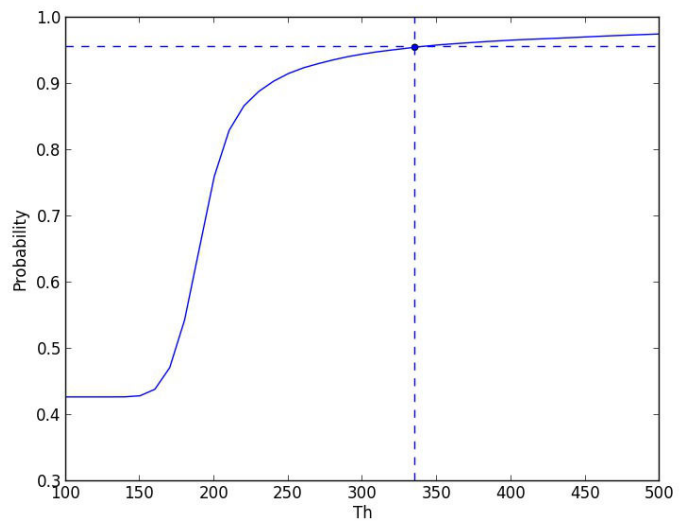

Figure 2. Probability for a period to be part an "activity". With a variability superior to $T h=335$, the probability is $95 \%$ to be correctly classified. This was computed on the 8 hours accelerometric signals collected from 15 subjects performing normal activities.

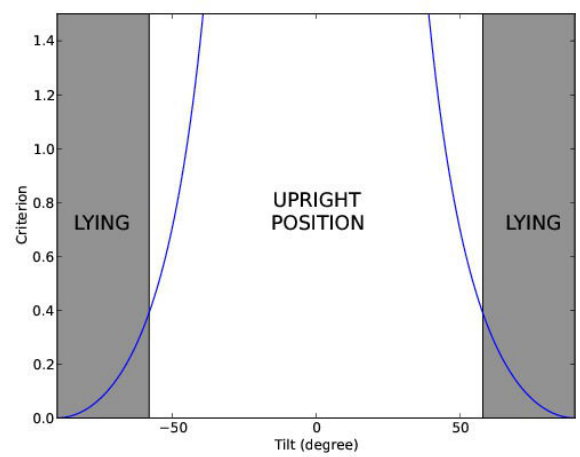

Figure 3. Our criterion of verticality shows a monotonic decreasing function to the tilt (absolute value).

Our criterion of verticality is maximal in upright position and minimal in the lying posture (Figure 3). It is therefore a criterion on the tilt, independent of the transversal plane orientation.

3) Detection of steps during walking

The detection of walking steps is obtained by triggering impacts on vertical acceleration signals [10]. The number of impacts (steps) are simply counted during a given interval of time. For instance, a walking speed $0.3-0.4 \mathrm{~m} / \mathrm{s}-$ average with COPD patients - will consist in 40 steps during one minute of walking. 


\section{RESULtS}

\section{A. Experimental Protocol}

The experiment was performed with 13 COPD patients (age $=64 \pm 7$ years, height $=168 \pm 10 \mathrm{~cm}$, weight $=75 \pm 17 \mathrm{~kg}$ ). The data profile (Figure 4) shows a homogeneous group of patients, mostly exhibiting a low level of activity.

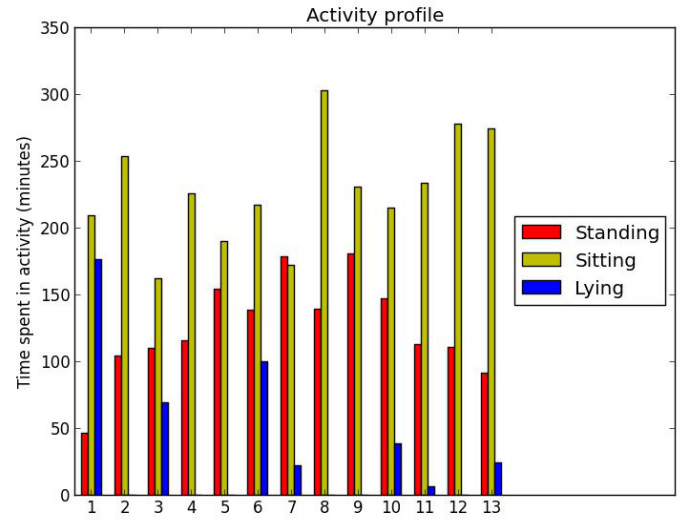

Figure 4. Data profile of the second experiment (involving 13 elderly COPD patients. The patients reported manually their activities.

The subjects had the IMU ACOR+ on their hip for 8 hours (from 9 am to $5 \mathrm{pm}$ ). They recorded manually - in a diary their real activities (i.e. walking, sitting for lunch, resting, etc.). As the self report is not precise enough and usually poorly estimates the duration of an activity [11], an expert performed a manual indexation of the recorded signals of acceleration with the help of the log file, to produce a reference.

\section{B. Comparison of episodes detections}

The outputs of our algorithm are further compared to the reference, episode by episode. We thus compute sensitivity and specificity. Sensitivity is the ratio between true positive (time correctly classified) against true positive plus false negative for the activity (the total time spent in this activity) found in the reference. Specificity is the ratio between true negative (time correctly classified as not doing the activity) against true negative plus false positive (the total time outside of this activity) according to the reference.

Table 1. Sensitivity and Specificity in detection of activities.

\begin{tabular}{l|l|l} 
Standing & Sitting & Lying
\end{tabular}

\begin{tabular}{|l|l|l|l|}
\hline Sensitivity & $94 \%$ & $87 \%$ & $77 \%$ \\
\hline Specificity & $86 \%$ & $91 \%$ & $90 \%$ \\
\hline
\end{tabular}

\section{A. Comparison of episodes durations}

To assess the accuracy of our classification process we investigated the similarity between the durations of activities (lying, sitting and standing) outputs of our algorithm with the reference durations of activity reported manually.

The correlations between the reported and computed activities are high (Table 2) for all static postures (standing, sitting and lying), even though the results include strong inter-patient variability. The standing posture is over evaluated because of the "tree classification algorithm" we used. The lower correlation for the time spent sitting is due to small lying time during daily activities.

Table 2. Correlation between temporal outputs

\begin{tabular}{|l|c|c|c|}
\hline & $\mathrm{R}$ & $\mathrm{P}$ & \\
\hline Standing & 0.89 & $<0.001$ & Pearson \\
\hline Sitting & 0.79 & 0.0012 & Pearson \\
\hline Lying & 0.92 & $<0.001$ & Spearman \\
\hline
\end{tabular}

For standing, we obtained a mean over-estimation of $23 \mathrm{~min}$ (Table 3). This is significant - p-value $<0.001$ - but this it is not correlated to the duration value $-\mathrm{p}$-value $>0.05$. The sitting durations shows a mean (negative) difference of 16 min - $p$-value $=0.06$. The difference of duration value between our algorithm and the reference are not correlated $p$-value $=0.95$. Concerning the lying posture, the median of the difference concluded with a null bias. The p-value from the Wilcoxon test is 0.14 , thus there is no significant mean bias for duration of lying. We observed one outlier, with a lying period largely under-estimated - 60 minutes instead of 145 minutes. Actually, it is due to a lying period incorrectly classified as sitting. The sensor was probably in inappropriate position or the subject was resting in a semireclining position.

We also analyzed the intra-class correlation coefficient (the ICC, Table 4). For standing and sitting, the ICC were over 0.71 , with no conclusion on the reproducibility. The reproducibility is good for the lying posture.

Table 3. Bland-Altman parameters for temporal outputs

\begin{tabular}{|l|c|c|c|}
\hline & $\begin{array}{c}\text { Mean } \\
\text { Difference }\end{array}$ & Correlation & $\mathrm{P}$ \\
\hline Standing & $23 \mathrm{~min}$ & 0.23 & Pearson/student \\
\hline Sitting & $-16 \mathrm{~min}$ & 0.019 & Pearson/student \\
\hline Lying & $0 \mathrm{~min}$ & -0.33 & Spearman/Wilcoxon \\
\hline
\end{tabular}


Table 4. IntraClass correlation of temporal outputs.

\begin{tabular}{|l|c|c|c|}
\hline & ICC & Confidence Interval & P \\
\hline Standing & 0.76 & $-0.02: 0.94$ & 0.19 \\
\hline Sitting & 0.75 & $0.35: 0.92$ & 0.07 \\
\hline Lying & 0.92 & $0.77: 0.97$ & $<0.001$ \\
\hline
\end{tabular}

\section{CONCLUSION}

We present our built-in algorithms embedded in our wearable IMU for for the automatic real time detection of postures. It extracts detailed characteristics of everyday physical activities - e.g. lying, sitting, standing and walkingtogether with corresponding energy expenditure.

We evaluated our algorithms with 13 volunteer COPD patients over 8 hours periods in everyday real-life situations. Our classification performances are good for detection of static postures with sensitivities 77-94\% and specificities from $86-91 \%$.

We demonstrate that activities can be classified in real time with embedded algorithms which are simple and robust. It potentially allows real time indexation of energy expenditure throughout the day; this is an important parameter for the monitoring of activities of COPD patients.

Our IMU has a built-in Bluetooth connectivity, which opens the way for real time data fusion with cardio-respiratory parameters obtained from other Bluetooth enabled sensors. For monitoring a patient in his daily life, the system must be used autonomously by the patient in his private environment. This implies to reduce the users' interactions which is obtained with the embedded intelligence. We must also minimise the impact of the device, with reduced form factors and enhanced autonomy.

\section{ACKNOWLEDGEMENTS}

The authors thank Anne Georges and Marion Lovato Henri Bazire pulmonary rehabilitation center (Saint Julien de Ratz, France), for supervision of data collection. We also wish to thank Sonia Dias-Domingos for statistical expertise of the temporal output parameters. This project was supported by the French Research Ministry under the grant number $1039 / 2010$

\section{REFERENCES}

[1] M. Decramer, W. Janssens, and M. Miravitlles, "Chronic obstructive pulmonary disease," The Lancet, 2012; 379 (9823): 1341-1351.

[2] F. Pitta, T. Troosters, M. A. Spruit, V. S. Probst, M. Decramer, and R. Gosselink, "Characteristics of physical activities in daily life in chronic obstructive pulmonary disease," Am J. Respir Crit Care Med, 2005; 171: 972-977.
[3] J. Garcia-Aymerich, E. Farrero, M. F'elez, J. Izquierdo, R. Marrades, and J. Anto, "Risk factors of readmission to hospital for a COPD exacerbation: a prospective study," Thorax, Feb. 2003; 58: 100-5.

[4] T. Troosters, F. Sciurba, S. Battaglia, D. Langer, S. R. Valluri, L. Martino, R. Benzo, D. Andre, I. Weisman, and M. Decramer, "Physical inactivity in patients with COPD, a controlled multi-center pilot-study," Respiratory Medicine, 2010; 104: 1005-1011.

[5] F. Sciurba, G. J. Criner, S. M. Lee, Z. Mohsenifar, D. Shade, W. Slivka, and R. A. Wise, "Six-minute walk distance in chronic obstructive pulmonary disease," American Journal of Respiratory and Critical Care Medicine, 2003; 167(11): 1522-1527.

[6] H. van Remoortel, Y. Raste, Z. Louvaris, S. Giavedoni, C. Burtin, D. Langer, F. Wilson, R. Rabinovich, I. Vogiatzis, N. S. Hopkinson, and T. Troosters, "Validity of six activity monitors in chronic obstructive pulmonary disease: a comparison with indirect calorimetry," PLoS ONE, Jun. 2012; 7(6): e39198.

[7] B. Perriot , J. Argod , J-L. Pepin and N. Noury, "A Network of Collaborative Sensors for the Monitoring of COPD Patients in their Daily Life", in proc 15th int. IEEE-Healthcom2013, pp.288-291.

[8] P. H. Veltink, H. B. Bussmann, W. de Vries, W. L. Martens, and R. C. V. Lummel, "Detection of static and dynamic activities using uniaxial accelerometers," IEEE TRNE, 1996; 4: 375-385.

[9] B. Perriot, J. Argod, J-L. Pepin and N. Noury, "Characterization of physical activity in COPD patients: validation of a robust algorithm for actigraphic measurements in ecological situation", IEEE-JBHI, 2014, Vol 18(4): 1225-1231.

[10] Najafi B, Aminian K, Paraschiv-Ionescu A, Loew F, Bula CJ, Robert P. Ambulatory system for human motion analysis using a kinematic sensor: monitoring of daily physical activity in the elderly. IEEETBME.2003;50(6): 711-723.

[11] F. Pitta, T. Troosters, V. S. Probst, M. A. Spruit, M. Decramer, and R. Gosselink, "Quantifying physical activity in daily life with questionnaires and motion sensors in COPD," European respiratory journal, 2006; 27(5): 1040-1055. 